\section{Persistência de hábitos de sucção não nutritiva: prevalência e fatores associados}

\section{Non-nutritive sucking habits persistence: prevalence and associated factors}

Maíra Pê Soares de Góes 1

Cláudia Marina Tavares Araújo 2 Paulo Sávio Angeiras Góes 3 Silvia Regina Jamelli 4

1-4 Pós-graduação em Saúde da Criança e do Adolescente. Centro de Ciências da Saúde. Universidade Federal de Pernambuco. Av. Professor Moraes Rego, 1235. Cidade Universitária. Recife, PE, Brasil. CEP: 50.670-901. E-mail: maira_goes@hotmail.com

\begin{abstract}
Objectives: to identify the prevalence of nonnutritive sucking habits in preschoolers and verify factors associated with persistent habit.

Methods: a cross-sectional analytical study conducted in 17 public educational units of Recife/PE through questionnaire with 524 preschooler guardians. Dependent (digital and pacifier sucking) and independent variables, related to the children (sex, age, breastfeeding, bottle feeding, nocturnal enuresis, school period, caregiver, birth order, dentist visit) and to their mothers (schooling, age, familiar income, outside work, work shift, cohabitation, habits guidance), were associated by Pearson's chi-square test, Fisher's Exact test and Poisson's regression.

Results: prevalence of nonnutritive sucking habits was 57\%; 47,5\% pacifier sucking, 5,7\% digital sucking and 3,8\% of both habits. After odds ratio adjustment, in a multivariate analysis, variables: breastfeeding and bottle feeding duration remained associated with pacifier sucking, while others variables as mother education, bottle feeding, and children's age and sex were statistically significant with digital sucking.

Conclusions: nonnutritive sucking habits showed high prevalence, being pacifier sucking most prevalent. Factors related to breastfeeding pattern (bottle feeding and breastfeeding duration) were explanatory factors to the habits persistence and psychosocial factors had relevant association.

Key words Pacifiers, Fingersucking, Habits
\end{abstract}

\section{Resumo}

Objetivos:identificar a prevalência de hábitos de sucção não nutritiva em pré-escolares e verificar fatores associados a sua persistência.

Métodos: estudo transversal e analítico realizado por meio de questionário com responsáveis de 524 crianças em 17 centros educacionais públicos de Recife/PE. Variáveis dependentes (sucção digital e de chupeta) e independentes, relacionadas à criança (sexo, idade, aleitamento materno, uso de mamadeira, enurese noturna, turnos na unidade educacional, cuidador, ordem de nascimento, visita ao dentista) e a sua mãe (escolaridade, idade, renda familiar, trabalho fora do domicilio, turnos de trabalho, coabitação, orientação sobre hábitos), foram associadas, usando os testes estatísticos qui-quadrado de Pearson, Exato de Fisher e regressão Poisson.

Resultados: prevalência de hábitos de sucção não nutritiva de 57\%: 47,5\% apenas sucção de chupeta. Após análise multivariada, as variáveis: tempo de aleitamento materno e uso de mamadeira continuaram associadas ao uso de chupeta, enquanto que as variáveis: escolaridade materna, uso de mamadeira, idade e sexo da criança encontraram-se fortemente associadas à sucção digital.

Conclusões: alta prevalência de hábitos de sucção não nutritiva, sendo a chupeta mais frequente. Aspectos associados ao padrão de aleitamento foram apontados como principais fatores explicativos à persistência destes hábitos em pré-escolares e os aspectos psicossociais tiveram poder de associação relevante.

Palavras-chave Chupetas, Sucção de dedo, Hábitos 


\section{Introdução}

A região mais importante do corpo da criança no seu primeiro ano de vida é a boca, pois por meio dela, o infante realiza a sucção. $\mathrm{O}$ ato de sugar é considerado um reflexo inato, observado antes mesmo do nascimento, na $29^{\circ}$ semana de vida intra-uterina e representa o padrão de comportamento mais primitivo e complexo do ser humano. A sucção é considerada a primeira atividade muscular coordenada da infância e do sistema estomatognático e tem sido reportada na literatura sob duas formas: nutritiva e não nutritiva. A sucção nutritiva ou fisiológica é fundamental para a sobrevivência dos neonatos e lactentes, já que instintivamente o conduz à satisfação de suas necessidades nutricionais. 1

A sucção não nutritiva é representada pelo hábito de sucção digital, de chupeta ou outro objeto, e usualmente proporciona à criança sensação de calor, bem-estar, prazer, segurança e proteção. ${ }^{2}$ No entanto, quando o hábito de sucção não nutritiva persiste por um período superior aos três anos de idade, ou seja, após a primeira infância, é considerado um hábito bucal deletério. 1

Os hábitos bucais deletérios estão fortemente associados com a presença de más oclusões, também denominadas de oclusopatias, e configuram-se, como um importante fator no desenvolvimento de alterações estruturais e funcionais do sistema estomatognático. Estas alterações podem afetar simultaneamente dentes, ossos, músculos e nervos, além de produzir problemas funcionais, estéticos ou esqueléticos nos dentes e/ou face. O surgimento desta má oclusão depende da intensidade, da frequência e da duração desse hábito, além da predisposição genética do indivíduo. 1,3

As oclusopatias, no Brasil, representam a terceira prioridade na escala de problemas de saúde bucal, perdendo apenas para a cárie e doença periodontal. No entanto, no Sistema Único de Saúde (SUS), não há tratamento efetivo para esses problemas de oclusão, apesar da ampla prevalência encontrada na população, como foi registrado no Saúde Bucal Brasil (SBBrasil 2010), um estudo realizado nas capitais brasileiras, no qual a prevalência de oclusopatias em crianças aos cinco anos de idade foi de $69,0 \% .4$

Diante desse contexto, observa-se a necessidade de medidas preventivas à ocorrência de oclusopatias na população. $\mathrm{O}$ conhecimento acerca da prevalência e dos fatores associados à presença destes hábitos deletérios na população infantil podem se tornar, portanto, um importante meio para elaboração destas medidas de prevenção, já que se constituem como um dos principais fatores etiológicos da má oclusão. Desta forma, o objetivo desse estudo foi identificar a prevalência de hábitos de sucção não nutritiva em crianças na idade pré-escolar, matriculadas em unidades educacionais públicas da cidade do Recife/PE, no Nordeste do Brasil e verificar os fatores associados a sua persistência.

\section{Métodos}

Foi realizado um estudo transversal com componente analítico em 17 unidades educacionais públicas da cidade de Recife-PE, região Nordeste do Brasil, no período de abril a dezembro de 2011, com mães ou responsáveis legais de crianças com idade entre três e cinco anos, de ambos os sexos. Crianças com malformações congênitas, incluindo fenda palatina e/ou labial, assim como, presença de alguma deficiência física ou mental, foram excluídas desta pesquisa.

A amostra foi calculada, considerando a prevalência de $40,0 \%$ de hábito de sucção não nutritiva,5 nível de confiança de $95,0 \%$ e erro máximo de $5,0 \%$, encontrando-se um tamanho estimado da amostra de 369 crianças. Como optou-se pelo cálculo de amostragem por conglomerado, sugere-se realizar a correção do efeito do desenho (deff) para correção do n. Assim, multiplicou-se o valor de $n$ obtido inicialmente por 1,5, resultando em 553. Para finalizar, foram acrescidos $10,0 \%$ para compensar eventuais perdas ao cálculo da amostra, totalizando 608 participantes.

A relação das unidades educacionais municipais foi obtida no endereço eletrônico da Secretaria de Educação, Esporte e Lazer da cidade do Recife. Foi registrado um total de 65 unidades educacionais municipais, que atendiam cerca de 16.450 crianças de zero a seis anos de idade. A partir dos números de telefone presentes na listagem, entrou-se em contato com as gestoras das unidades educacionais para obtenção da quantidade de crianças matriculadas em cada unidade. $\mathrm{Na}$ faixa etária de interesse da pesquisa, existiam cerca de 3200 crianças distribuídas em 61 unidades educacionais municipais. Ressalta-se que quatro unidades estavam desativadas por ocasião deste recrutamento, sendo, portanto, excluídas da pesquisa.

Diante deste contexto, a seleção da amostra foi realizada por processo de amostragem por conglomerados, em duas etapas. Inicialmente realizou-se o sorteio aleatório dos conglomerados, neste caso representado pelos centros educacionais, e em seguida o sorteio aleatório dos indivíduos, isto é, os pré-escolares. Do universo de 61 unidades educa- 
cionais disponíveis para a pesquisa, 17 foram sorteadas aleatoriamente, de forma que unidades de ensino de todas as seis regiões político administrativas da cidade fossem abordadas. E, por fim, foi realizado o sorteio aleatório das crianças a serem entrevistadas em cada unidade educacional, da forma que o número de alunos, por centro educacional, foi determinado proporcionalmente ao número total de alunos, na faixa etária de interesse, em cada unidade de ensino.

As entrevistas foram realizadas nas próprias unidades educacionais no horário de chegada ou de saída das crianças, por meio de um questionário padronizado e estruturado, com seus pais ou responsáveis legais. Neste questionário, foram colhidas informações sobre a criança, como: idade $(3,4$ ou 5 anos), sexo (masculino ou feminino), turno na unidade educacional (tempo que permaneciam no centro educacional: um turno ou integral), enurese noturna (presença ou ausência na época da pesquisa), ordem de nascimento (primogênito; filho do meio; caçula), cuidador (quem é o principal cuidador da criança: pai/mãe; outra pessoa da família; unidade escolar), visita ao dentista (se a criança já foi ao dentista anteriormente: sim ou não), sucção de chupeta e/ou de dedo (presença ou ausência; tempo do hábito: $<36$ meses de idade; $\geq 36$ meses de idade), aleitamento materno (por quanto tempo: 0-3 meses; $4-6$ meses; $>6$ meses) e uso de mamadeira (sim ou não).

As perguntas continham também questionamentos sobre as mães das crianças, tais como: escolaridade ( $\leq 8$ anos de estudo; 9 a 11 anos de estudo; $\geq 12$ anos de estudo), turno de trabalho (nenhum turno; um turno; dois ou mais turnos), trabalho fora do domicílio (sim ou não), idade ( $\leq 24$ anos; 25 - 29 anos; $\geq 30$ anos), coabitação (com companheiro ou sem companheiro) e renda familiar $(<1$ salário mínimo ou $\geq 1$ salário mínimo).

A variável de desfecho foi representada pela presença do hábito de sucção de chupeta ou de dedo pela criança, no presente ou no passado. A variável foi categorizada de acordo com a duração do hábito em: menos de 36 meses ou mais de 36 meses. Essa diferenciação foi estabelecida de acordo com relatos da literatura, os quais consideram que a maioria das crianças deveria abandonar o hábito de sucção não nutritivo antes dos três anos de idade, uma vez que a persistência desse hábito após a primeira infância pode representar uma regressão ao comportamento infantil. A idade limite de cinco anos foi estabelecida porque a manutenção do hábito a partir desta idade pode causar alterações permanentes na oclusão dentária da criança, além de interferir no padrão de desenvolvimento craniofacial e provocar alterações no sistema estomatognático. 5,6

O processamento dos dados realizou-se por meio de dupla entrada para avaliação da consistência da digitação utilizando-se o software Epi-Info 6.04. Posteriormente, estes dados foram analisados com o emprego do software Statistical Package for the Social Sciences (SPSS), versão 17.

Utilizou-se o teste do qui-quadrado de Pearson ou o Exato de Fisher, quando indicado, como testes de significância na comparação das variáveis categóricas. A razão de prevalência (RP) foi utilizada como medida de associação nas análises bivariadas com seus respectivos intervalos de confiança de $95 \%$. Definiu-se como categoria de referência aquela que teoricamente apresentasse menor risco esperado para os desfechos. Adotou-se o valor de $p<0,05$ como estatisticamente significante.

Utilizou-se a análise de regressão de Poisson a fim de ajustar os possíveis fatores de confundimento relacionados ao uso da chupeta e à sucção digital, adotando-se a abordagem hierarquizada de entrada das variáveis nos modelos de regressão. As variáveis com valor de $p<0,20$ na análise bivariada foram selecionadas para inclusão na análise de regressão múltipla utilizando-se o pacote estatístico Stata versão 11 .

Inicialmente, as variáveis selecionadas para serem introduzidas na análise de regressão em relação ao uso da chupeta foram: escolaridade materna e cuidador da criança. No segundo bloco introduziram-se tempo de amamentação e uso de mamadeira e, no terceiro e último bloco, turno de frequência da criança na unidade educacional e ocorrência de enurese noturna. Para a análise de regressão com a sucção digital como desfecho, incluíram-se no primeiro bloco a escolaridade materna e renda familiar. No segundo bloco introduziu-se o uso de mamadeira, sexo e idade da criança e no terceiro e último bloco o turno de frequência da criança na unidade educacional. A partir do segundo bloco, as análises foram ajustadas pelas variáveis dos blocos anteriores, por meio do uso do software Epi-Info 6.04

Este estudo foi previamente analisado e aprovado pelo Comitê de Ética em Pesquisa com seres humanos da Universidade Federal de Pernambuco (UFPE), conforme protocolo $n^{\circ} 017 / 11$ e CAAE $n^{\circ} 0498.0 .12 .000 / 11$. Todos os participantes assinaram termo de consentimento livre e esclarecido. 


\section{Resultados}

Durante o período de pesquisa, 524 mães ou responsáveis legais de crianças de ambos os sexos, com idade entre três e cinco anos, foram entrevistadas e consideradas válidas para a pesquisa. Não obstante, o cálculo amostral resultou em 553 sujeitos, intercorrências durante a coleta de dados impossibilitaram o alcance do total de entrevistados. Dessa forma, ocorreram 29 perdas, as quais representaram apenas $5,24 \%$ da amostra ideal.

Das crianças entrevistadas, a maioria apresentava idade entre três e quatro anos $(71,2 \%)$ e existiam 274 (52,3\%) meninos e $250(47,7 \%)$ meninas (Tabela 1). A prevalência de hábitos de sucção não nutritiva foi de 58,2\% (IC 95\%: 54,0\% - 62,4\%), sendo $48,7 \%$ apenas por uso de chupeta, $5,7 \%$ apenas por sucção digital e $3,8 \%$ por ambos os hábitos.

Com relação às características dos pré-escolares, foram observadas diferenças estatisticamente significantes na análise univariada com cada variável de desfecho. O hábito de sucção digital foi mais frequente em meninas $(p=0,006)$ com cinco anos de idade $(p=0,005)$ que usavam mamadeira $(p=0,04) \mathrm{e}$ frequentavam a unidade de ensino por apenas um turno ( $p=0,004)$. Quanto ao uso de chupeta, este hábito foi mais frequente nos pré-escolares que estudavam em turno integral $(p=0,02)$ e apresentavam enurese noturna $(p=0,03)$, como também nas crianças que tiveram aleitamento materno por um período menor que quatro meses $(p<0,001)$ e usavam mamadeira $(p<0,001)$ (Tabela 1$)$.

A respeito das características maternas e familiares, foi observado que as famílias entrevistadas pertenciam a um nível socioeconômico baixo, visto que $2 / 3$ da amostra apresentavam renda mensal menor que um salário mínimo brasileiro e a maioria das mães haviam estudado por um período menor do que oito anos. A escolaridade materna foi o único fator socioeconômico associado com a presença do hábito de sucção não nutritiva, especialmente a sucção digital, pois em relação à sucção de chupeta, esta associação foi limítrofe $(p=0,05)$ com mães cujo tempo de estudo não ultrapassou oito anos. Por sua vez, houve associação estatisticamente significante na presença de sucção digital em crianças cujas mães apresentavam doze anos ou mais de estudo ( $p=0,02)$ (Tabela 2).

As Tabelas 3 e 4 apresentam, para os hábitos de sucção de chupeta e digital, respectivamente, os resultados da análise de Poisson, após o ajuste das razões de prevalência.

Para a presença do hábito de sucção de chupeta em pré-escolares, as variáveis que permaneceram no modelo final ajustado foram àquelas referentes ao Bloco 2: tempo de aleitamento materno inferior a três meses $(p<0,001)$ e uso de mamadeira presente $(p<0,001)$. A enurese noturna $(p=0,37)$ e o tempo de permanência na unidade de ensino $(p=0,20)$, do Bloco 3, perderam o efeito, ou seja, não mostraram significância (Tabela 3).

Em relação ao hábito de sucção digital em préescolares, permaneceram no modelo final ajustado, as variáveis referentes ao Bloco 2: idade de cinco anos $(p=0,02)$, sexo feminino $(p=0,03)$ e uso de mamadeira $(p=0,02)$. Dentre as variáveis do Bloco 1 , apenas a escolaridade materna com no mínimo doze anos de estudo $(p=0,02)$ continuou como fator de risco independente das outras variáveis. O tempo de permanência na unidade de ensino $(p=0,08)$, do Bloco 3, perdeu o efeito, ou seja, não mostrou significância (Tabela 4).

\section{Discussão}

O hábito de sucção não nutritiva é um assunto de grande interesse para diferentes profissionais da área da saúde, pois causa alterações no sistema estomatognático, além de estar diretamente relacionado ao comportamento da criança e da sua família. Dessa forma, o conhecimento da prevalência e dos fatores associados à sua instalação e persistência, adquire uma importância fundamental.

A prevalência de hábitos de sucção não nutritivos nas crianças pré-escolares pesquisadas $(58,2 \%)$ foi um pouco acima do valor encontrado nos últimos estudos realizados no país, em 2009,5 20117 e 2013,8 nos quais a prevalência foi de $40,0 \%$ $43,5 \%$ e $53,3 \%$, respectivamente. Estes valores apontam para um aumento na prevalência destes hábitos entre as crianças, como provável consequência do forte componente cultural e social envolvido no problema: a oferta da chupeta e o padrão de aleitamento.

A sucção de chupeta foi o hábito de sucção não nutritiva mais prevalente no estudo, e representa um costume bastante arraigado na cultura tanto do Brasil quanto de outros países de cultura ocidental.6,9-14 A chupeta constitui um bem de consumo de preço reduzido, acessível à população, e com sua utilização amplamente estimulada pelos pais e cuidadores infantis, frente ao choro infantil, com o objetivo de acalmar e reconfortar, 15 apesar de seu uso ser desaconselhado pela Organização Mundial da Saúde (OMS). 16

A OMS incentiva a prática do aleitamento 


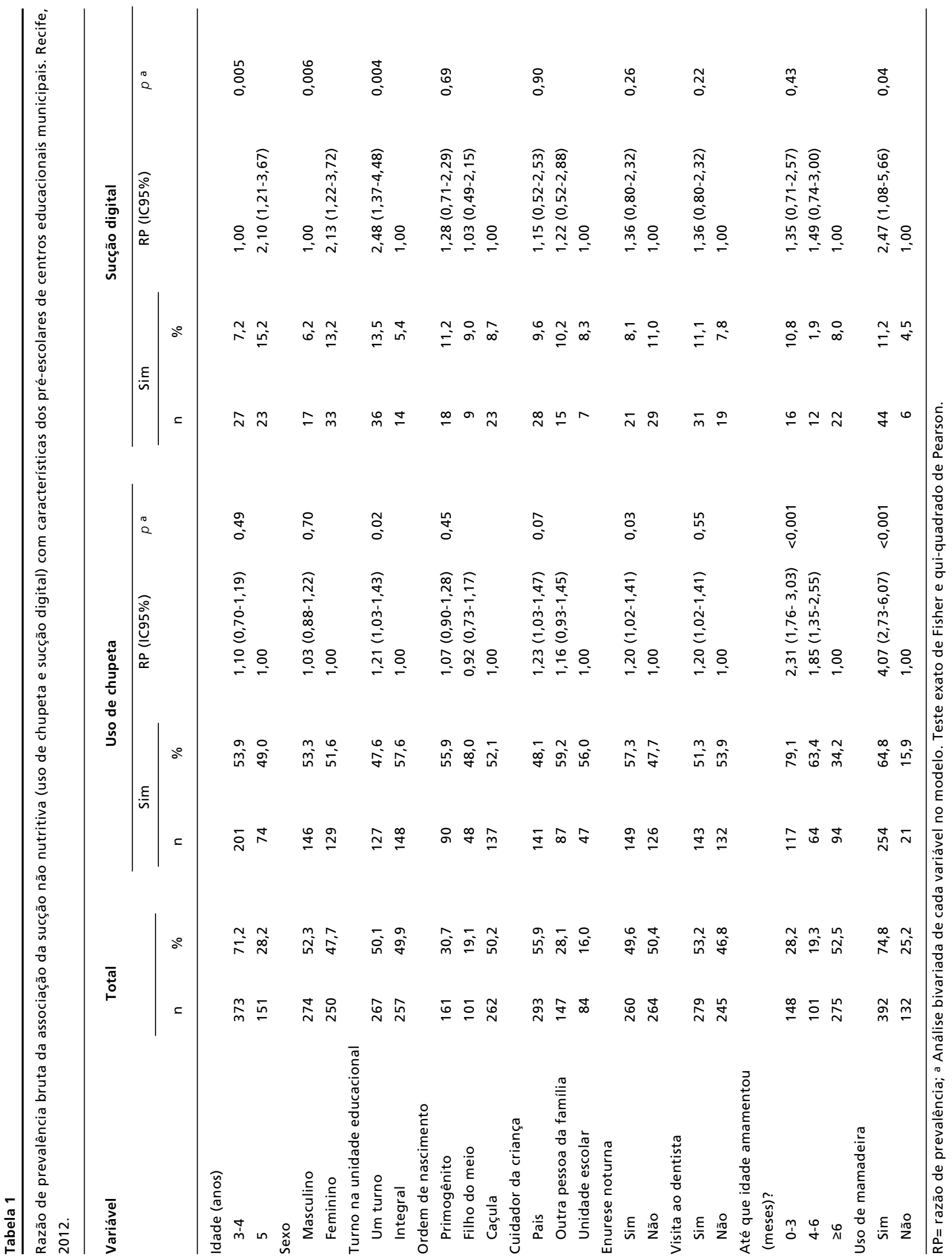




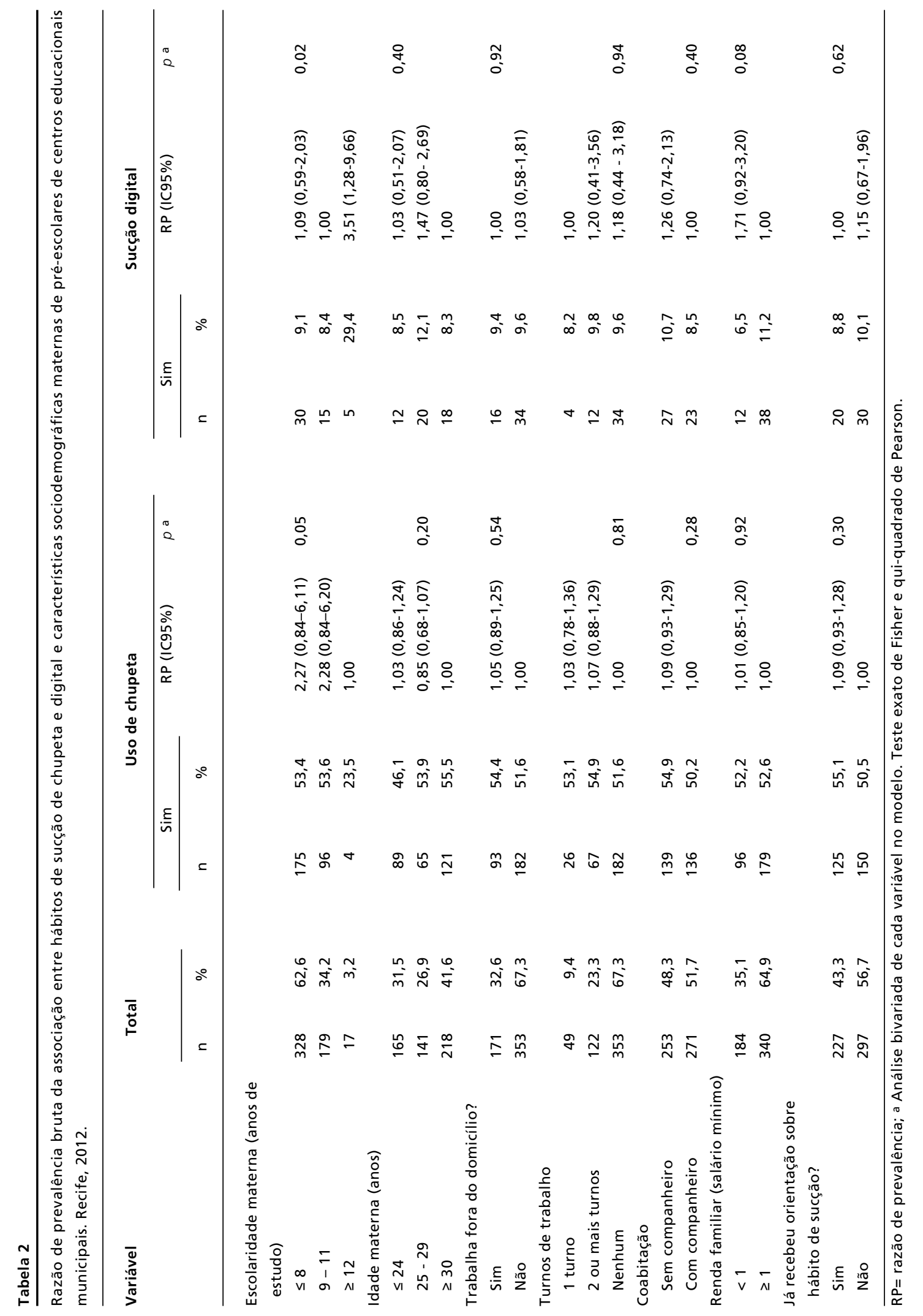




\section{Tabela 3}

Razão de prevalência bruta e ajustada dos fatores associados ao uso de chupeta em pré-escolares de centros educacionais municipais. Recife, 2012.

\begin{tabular}{|c|c|c|c|c|}
\hline Variável & RP bruta (IC95\%) & $p$ & RP ajustada (IC95\%) & $p$ a \\
\hline \multicolumn{5}{|l|}{ Bloco 1} \\
\hline \multicolumn{5}{|c|}{ Escolaridade da mãe (anos de estudo) } \\
\hline$\leq 8$ & $2,27(0,84-6,11)$ & & $2,26(0,84-6,08)$ & 0,11 \\
\hline $9-11$ & $2,28(0,84-6,20)$ & & $2,34(0,85-6,36)$ & 0,10 \\
\hline$\geq 12$ & 1,00 & 0,05 & & \\
\hline \multicolumn{5}{|l|}{ Cuidador da criança } \\
\hline Outro familiar & $1,23(1,03-1,47)$ & & $1,24(0,95-1,62)$ & 0,12 \\
\hline Outra pessoa/escola & $1,16(0,93-1,45)$ & & $1,18(0,84-1,63)$ & 0,35 \\
\hline Pais & 1,00 & 0,07 & & \\
\hline \multicolumn{5}{|l|}{ Bloco 2} \\
\hline \multicolumn{5}{|c|}{ Tempo de amamentação (meses) } \\
\hline $0-3$ & $2,31(1,76-3,03)$ & & $1,66(1,25-2,21)$ & $<0,001$ \\
\hline $4-6$ & $1,85(1,35-2,55)$ & & $1,37(0,99-1,91)$ & 0,06 \\
\hline$>6$ & 1,00 & $<0,001$ & & \\
\hline \multicolumn{5}{|l|}{ Uso de mamadeira } \\
\hline $\operatorname{Sim}$ & $4,07(2,73-6,07)$ & & $3,15(1,97-5,05)$ & $<0,001$ \\
\hline Não & 1,00 & $<0,001$ & & \\
\hline \multicolumn{5}{|l|}{ Bloco 3} \\
\hline \multicolumn{5}{|c|}{ Turnos na unidade educacional } \\
\hline Integral & $1,21(1,03-1,43)$ & & $1,19(0,91-1,54)$ & 0,20 \\
\hline Manhã ou tarde & 1,00 & 0,02 & & \\
\hline \multicolumn{5}{|l|}{ Enurese noturna } \\
\hline Sim & $1,20(1,02-1,41)$ & & $1,12(0,88-1,42)$ & 0,37 \\
\hline Não & 1,00 & 0,03 & & \\
\hline
\end{tabular}

$\mathrm{RP}=$ razão de prevalência; a Análise multivariada de cada variável no modelo. Regressão logística de Poisson. 
Razão de prevalência bruta e ajustada e respectivos intervalos de confiança para os fatores associados à sucção digital em pré-escolares de centros educacionais municipais do Recife, 2012.

\begin{tabular}{|c|c|c|c|c|}
\hline Variável & RP bruta (IC95\%) & $p$ & RP ajustada (IC95\%) & $p$ a \\
\hline \multicolumn{5}{|l|}{ Bloco 1} \\
\hline \multicolumn{5}{|c|}{ Escolaridade da mãe (anos de estudo) } \\
\hline$\leq 8$ & $1,09(0,59-2,03)$ & & $1,20(0,64-2,24)$ & 0,58 \\
\hline $9-11$ & 1,00 & 0,02 & & \\
\hline$\geq 12$ & $3,51(1,28-9,66)$ & & $3,27(1,19-9,03)$ & 0,02 \\
\hline \multicolumn{5}{|c|}{ Renda familiar (salário mínimo) } \\
\hline$\geq 1$ & $1,71(0,92-3,20)$ & & $1,64(0,84-3,20)$ & 0,15 \\
\hline$<1$ & 1,00 & 0,08 & & \\
\hline \multicolumn{5}{|c|}{ Bloco 2} \\
\hline \multicolumn{5}{|c|}{ Idade da criança (anos) } \\
\hline 5 & $2,10(1,21-3,67)$ & & $2,01(1,13-3,58)$ & 0,02 \\
\hline $3-4$ & 1,00 & 0,005 & & \\
\hline \multicolumn{5}{|l|}{ Sexo da criança } \\
\hline Feminino & $2,13(1,22-3,72)$ & & $1,96(1,08-3,53)$ & 0,03 \\
\hline Masculino & 1,00 & 0,006 & & \\
\hline \multicolumn{5}{|l|}{ Uso de mamadeira } \\
\hline Sim & $2,47(1,08-5,66)$ & & $2,78(1,18-6,57)$ & 0,02 \\
\hline Não & 1,00 & 0,04 & & \\
\hline \multicolumn{5}{|l|}{ Bloco 3} \\
\hline \multicolumn{5}{|c|}{ Turnos na unidade educacional } \\
\hline Manhã ou tarde & $2,48(1,37-4,48)$ & & $1,97(0,93-4,17)$ & 0,08 \\
\hline Integral & 1,00 & 0,004 & & \\
\hline
\end{tabular}

materno devido a seus reconhecidos benefícios nutricional, imunológico, cognitivo, econômico e social e não recomenda o uso da chupeta, especialmente em crianças amamentadas naturalmente, para evitar a confusão de bicos e o desmame precoce. ${ }^{7,16} \mathrm{~A}$ amamentação natural é considerada um fator de proteção para persistência do hábito de sucção de chupeta, haja vista a prevalência de este hábito ter-se reduzido na medida em que o tempo de aleitamento materno foi maior. Crianças amamentadas naturalmente são menos propensas a persistir com hábitos de sucção não nutritivos, corroborando os resultados de outros estudos. $2,7,8,11,17-19$

$\mathrm{O}$ aleitamento materno promove um intenso trabalho da musculatura facial, influencia o desenvolvimento ósseo e muscular, gerando fadiga nos músculos, fazendo com que a criança satisfaça seu instinto de sugar e não necessite de uma sucção não nutritiva, ${ }^{1}$ ou seja, supre tanto a necessidade de sucção nutritiva como a não nutritiva e, por esta razão, a criança não recorre a estímulos artificiais de sucção, como a chupeta. Além disso, a amamentação natural tem efeitos positivos sobre o desenvolvimento infantil psicológico e, ainda, sobre o sistema estomatognático por ser um estimulante do crescimento natural e normal ortopédico dos maxilares. 8 Quando ocorre a substituição do aleitamento materno pela mamadeira, o lactente não fica satisfeito porque nem o bico utilizado na mamadeira é adequado, nem sua musculatura orofacial fica cansada o suficiente, uma vez que a amamentação artificial pelo uso da mamadeira promove o estímulo apenas dos músculos bucinadores e do orbicular da boca, deixando de utilizar outros músculos faciais, interferindo no crescimento craniofacial. Induz, ainda, a alterações na mastigação, deglutição e fonação, podendo conduzir a oclusopatias. ${ }^{20}$

O uso da mamadeira, portanto, representa um fator de risco tanto para o uso persistente da chupeta como da sucção digital, haja vista sua significantemente associação com ambos os hábitos. Desta forma, conclui-se que o uso da mamadeira, independentemente do tempo de utilização, pode representar uma maior chance de a criança manter o hábito de 
sucção não nutritiva em relação àquelas que nunca a utilizaram. ${ }^{11,19}$ Portanto, é possível inferir que a prática de aleitamento materno prolongado sem uso de mamadeiras e bicos artificiais, parece exercer efeitos muito positivos na prevenção de hábitos de sucção não nutritiva. ${ }^{7}$

No presente estudo, mais da metade das crianças $(52,5 \%)$ eram ou foram usuárias de chupeta e deste total, $28,6 \%$ a usaram durante 36 meses ou mais de idade, isto é, um pouco mais da metade das crianças que iniciaram o hábito, persistiram com ele após a primeira infância (54,5\%). Em relação ao hábito de sucção digital foi verificado que das 50 crianças que iniciaram o hábito, 45 (90,2\%) permaneceram com ele por pelo menos 36 meses de idade. Esses resultados apontam para uma baixa prevalência do habito de sucção digital, mas também, para uma maior dificuldade na remoção deste hábito quando instalado.

Diante desse contexto, os nossos resultados suscitam a discussão acerca da oferta da chupeta quando se percebe que a criança tende a persistir com o hábito de sucção digital, em especial para aquelas que iniciam o hábito ainda na vida intrauterina. A introdução do uso racional da chupeta, apenas nos momentos de necessidade de sucção não nutritiva, os quais normalmente ocorrem após a amamentação, até no máximo 24 meses de vida, pode ser considerada uma alternativa plausível para evitar que a criança persista com o hábito de sucção digital. 21

Além disso, os resultados encontrados apontaram para uma tendência decrescente do uso da chupeta com o aumento da idade das crianças em oposição ao hábito de sucção digital que se mostrou em ascendência, ratificando que a sucção digital é um hábito mais acessível para a criança quando comparado à chupeta e, por isso, sua interrupção sofre maiores dificuldades, e o torna um hábito mais propenso a persistir até a idade pré-escolar e escolar. ${ }^{22}$ No presente estudo, a idade de cinco anos permaneceu como fator de risco independente das demais variáveis para a persistência da sucção digital. Resultados semelhantes foram encontrados tanto em países ocidentais quanto em orientais, tais como Reino Unido, 13,22 Arábia Saudita 23 e também em diferentes regiões do Brasil.24,25

A despeito de alguns estudos relatarem não haver associação entre sexo e prevalência de hábito de sucção não nutritiva, $6,23,26$ nesta pesquisa, a sucção digital foi mais frequente em meninas, com significância estatística, permanecendo como fator de risco independente das demais variáveis, no modelo final ajustado. Outros trabalhos encontraram resultados semelhantes e apontam para a existência de fatores culturais que diferenciam os gêneros. $5,24,27$

A sucção digital não apresentou associação significante com o aleitamento materno, corroborando resultados de outras pesquisas. ${ }^{18,24,28}$ Esse hábito apresentou baixa prevalência em todos os estudos, o que pode ter conduzido à não associação entre as variáveis. Em contrapartida, em países de cultura africana, a sucção digital normalmente é apontada como o hábito de sucção não nutritiva mais prevalente. 29,30

No presente estudo, a renda familiar não foi associada à presença de hábitos de sucção não nutritiva. Este fato é uma provável consequência da elevada homogeneidade quanto às características socioeconômicas da população estudada, na qual a maioria era pertencente ao estrato classificado como de baixa renda.

Farsi e Salama 23 constataram que a industrialização e a modernização da sociedade requer a participação das mulheres no mercado de trabalho, o que causa a diminuição na duração do aleitamento materno e provoca nas crianças uma maior adoção de hábitos de sucção digital e de chupeta, concluindo que quanto maior o nível de escolaridade dos pais, maior a porcentagem de crianças em idade préescolar que utilizam a chupeta. No presente estudo, a associação positiva entre presença de hábito de sucção não nutritiva e maior nível de escolaridade, foi encontrada entre a sucção digital e o maior nível de escolaridade materna.

A prevenção na instalação e persistência dos hábitos de sucção não nutritiva representa uma política de saúde pública relevante no que diz respeito à prevenção de oclusopatias, um problema de saúde pública com prevalência de $69,0 \%$ em crianças de cinco anos de idade no Brasil, segundo fonte do SBBrasil 2010.4 Outros dados do Ministério da Saúde ${ }^{9}$ também mostraram relação entre padrão de aleitamento e o uso de chupeta e, consequentemente, maior presença de oclusopatias. Segundo estes dados, na Região Norte do Brasil, onde há maior concentração de populações indígenas, amamenta-se mais, e encontram-se menores índices de uso de chupeta e mamadeira, além de menor prevalência de oclusopatias. Em regiões como Sul e Sudeste, sendo a necessidade da mulher no mercado de trabalho é maior, registram-se redução no tempo ou duração de aleitamento materno e maior frequência no uso de mamadeira e chupeta e, ainda, aumento na prevalência de oclusopatias.

Algumas limitações podem ser evidenciadas neste estudo. Durante a coleta de dados, não foi possível realizar o reexame dos membros da 
amostra, problema que pode ter sido minimizado por existir apenas um examinador e pelo número de indivíduos entrevistados ter sido alto, diminuindo assim os erros de uma baixa concordância. Além disso, os resultados devem ser explorados com cautela, pois a população estudada foi limitada a famílias de baixa renda, que frequentavam unidades educacionais municipais, o que não garante sua reprodução em outras realidades socioeconômicas e culturais. A possibilidade do viés de memória é provável, apesar de a informação coletada ter acontecido durante ou pouco tempo depois da exposição. Por fim, a baixa prevalência do hábito de sucção digital na população estudada pode ter impedido associação significante com algumas variáveis psicossociais investigadas.

Os achados deste estudo demonstram ser essencial orientar pais e responsáveis acerca da importância da amamentação natural como fator de proteção e do uso de mamadeira como fator de risco para instalação e persistência destes hábitos. Dessa forma, aspectos referentes à prevenção de hábitos bucais deletérios, principalmente sucção digital e uso de chupeta, devem ser considerados de forma mais enfática nas práticas e políticas de saúde já existentes, tanto nos trabalhos e cursos de promoção e incentivo ao aleitamento materno, especialmente para gestantes, como em medidas educativas, realizadas em unidades de educação e de saúde com pais e profissionais. Assim, será possível diminuir a

\section{Referências}

1. Casagrande L, Ferreira FV, Hahn D, Unfer DT, Praetzel JR. Aleitamento natural e artificial e odesenvolvimento do sistema estomatogmático. Rev Fac Odontol Porto Alegre. 2008; 49 (2): 11-17.

2. Ngom PI, Diagne F, Samba Diouf J, Ndiaye A, Hennequin M. Prevalence and factors associated with non-nutritive sucking behavior. Cross sectional study among 5- to 6-yearold Senegalese children. L'Orthodontie Française. 2008; 79 (2): 99-106.

3. Boeck EM, Pizzol KEDC, Barbosa EGP, Pires NCA, Lunardi N. Prevalência de má oclusão em crianças de 3 a 6 anos portadoras de hábito de sucção de dedo e/ou chupeta Rev Odontol UNESP. 2013; 42(2): 110-116.

4. Brasil. Ministério da Saúde. Projeto SB Brasil 2010 Pesquisa Nacional de Saúde Bucal - Resultados principais. Brasília, DF; 2011

5. Santos SA, Holanda ALF, Sena MF, Gondim LAM, Ferreira MAF. Non-nutritive sucking habits among preschool-aged children. J Pediatr. 2009; 85 (5): 408-14.

6. Warren JW, Bishara SE, Steinbock KL, Yonezu T, Nowak AJ. Effects of oral habits' duration on dental characteristics in the primary dentition. J Am Dent Assoc. 2001; 132 (12): 1685-93.

7. Vasconcelos FMN, Massoni ACLT, Heimer MV, Ferreira prevalência das oclusopatias, um problema de saúde pública brasileiro.

Por fim, os dados expostos permitem concluir que a prevalência dos hábitos de sucção não nutritiva em crianças na faixa etária entre três e cinco anos foi alta, sendo o hábito de sucção de chupeta mais prevalente, e que a pouca duração do aleitamento materno foi a principal justificativa encontrada para a persistência de hábitos de sucção não nutritiva em pré-escolares. Desta forma, o aleitamento materno por seis meses ou mais, quando não associado ao uso de mamadeiras e bicos artificiais, pode ser considerado um método de excelência na prevenção de hábitos de sucção não nutritiva.

\section{Agradecimentos}

À Professora Marilia de Carvalho Lima do Departamento Materno-Infantil da Universidade Federal de Pernambuco e Coordenadora do Programa de Pós Graduação em Saúde da Criança e do Adolescente, pelos comentários e sugestões durante a análise dos dados deste artigo. Ao CNPq, pela aprovação do projeto no Edital/Chamada Universal 14/2011, processo número 475202/2011, do qual a maioria dos autores é colaborador, visto que meu projeto de pesquisa serviu como base juntamente com outros projetos.

AMB, Katz CRT, Rosenblat A. Non-nutritive sucking habits, anterior open bite and associated factors in Brazilian children aged 30-59 months. Braz Dent J. 2011; 22 (2): $140-5$.

8. Moimaz SAS, Rocha NB da, Garbin AJI, Saliba O. A influência da prática do aleitamento materno na aquisição de hábitos de sucção não nutritivos e prevenção de oclusopatias. Rev. odontol. UNESP. 2013; 42 (1): 31-36

9. Brasil. Ministério da Saúde. Pesquisa de prevalência do aleitamento materno nas capitais e no Distrito Federal. Brasília, DF; 2001.

10. Katz CRT, Rosenblatt A, Colares V. Hábitos de sucção, onicofagia e enurese noturna em pré-escolares do RecifePE. JBP J Bras Odontopediatr Odontol Bebê. 2004; 7 (37): 258-65.

11. Leite-Cavalcanti A, Medeiros-Bezerra PK, Moura C. Aleitamento natural, aleitamento artificial, hábitos de sucção e maloclusões em pré-escolares brasileiros. Rev Salud Pública. 2007; 9 (2): 194-204.

12. Peres KG, Barros AJD, Peres MA, Victora CG. Effects of breastfeeding and sucking habits on malocclusion in a birth cohort study. Rev Saúde Pública. 2007; 41 (3): 343-50.

13. Duncan K, Mcnamara C, Ireland AJ, Sandy Jr. Sucking habits in childhood and the effects on the primary dentition: 
findings of the Avon Longitudinal Study of Pregnancy and Childhood. Int J Paediatr Dent. 2008; 18 (3): 178-88.

14. Dimberg L, Bondemark L, Söderfeldt B, Lennartsson B Prevalence of malocclusion traits and sucking habits among 3-year-old children. Swed Dent J. 2010, 34 (1): 35-42.

15. Tomita NE, Sheiham A, Bijella VT, Franco LJ. Relação entre determinantes socioeconômicos e hábitos bucais de risco para más-oclusões em pré-escolares. Pesq Odontol Bras. 2000, 14 (2): 169-175.

16. WHO (World Health Organization). Report of the expert consultation on the optimal duration of exclusive breastfeeding: conclusions and recommendations. Geneva; 2001.

17. Telles FBA, Ferreira RI, Magalhães LNC, Scavone-Junior $\mathrm{H}$. Effect of breast- and bottle-feeding duration on the age of pacifier use persistence. Braz Oral Res. 2009; 23 (4): 432-8.

18. Scavone-Junior H, Guimarães-Junior $\mathrm{CH}$, Ferreira RI, Nahas AC, Vellini-Ferreira F. Association between breastfeeding duration and non-nutritive sucking habits. Community Dent Health. 2008; 25: 161-5.

19. Bueno SB, Bittar TO, Vazquez FL, Meneghim MC, Pereira AC. Association of breastfeeding, pacifier use, breathing pattern and malocclusions in preschoolers. Dental Press J Orthod. 2013; 18 (1): 301-6.

20. Carvalho GD. Amamentação e prevenção das alterações funcionais e estruturais do sistema estomatognático. Odontol Ens Pesq. 1998; 2 (1): 339-48.

21. Scavone-Junior H, Ferreira RI, Mendes TE, Vellini-Ferreira F. Prevalence of posterior crossbites among pacifier users: a study in the deciduous dentition. Braz Oral Res. 2007; 21: 153-8.

22. Patel A, Moles D, O'nei, J, Noar J. Digit sucking in children resident in Kettering (UK). J Orthod. 2008; 35: 25561 .

Recebido em 2 de outubro de 2012

Versão final apresentada em 5 de abril de 2013

Aprovado em 20 de junho de 2013
23. Farsi NM, Salama FS. Sucking habits in Saudi children: prevalence, contributing factors and effects on the primary dentition. Pediatr Dent. 1997; 19: 28-33.

24. Holanda ALF, Santos SA, Sena MF, Ferreira MAF. Relationship between breast and bottle-feeding and nonnutritive sucking habits. Oral Health Prev Dent. 2009; 7: $331-7$.

25. Pizzol KERC, Boeck EM, Santos LFP, Lunardi N, Oliveira JPL. Influência do ambiente familiar e da condição socioeconômica na introdução e na manutenção de hábito de sucção não nutritiva. Rev Odontol UNESP. 2011; 40 (6): 296-303.

26. Bosnjak A, Vucicevic-Boras V, Miletic I, Bozic D, Vukelja $M$. Incidence of oral habits in children with mixed dentition. J Oral Rehabil. 2002; 29: 902-5.

27. Magalhaes L do NC, Rodrigues MJ, Heimer MV, Alencar AS de. Prevalence of non-nutritive sucking habits and its relation with anterior open bite in children seen in the Odontopediatric Clinic of the University of Pernambuco. Dental Press J. Orthod. 2012; 17 (2): 119-23.

28. Jahanbin A, Mokhber N, Jabbarimani A. Association between socio demographic factors and nutritive and nonnutritive sucking habits among Iranian girls. East Mediterr Health J. 2010; 16 (11): 1143-7.

29. Quashie-Williams R, Costa OO, Isiekwe MC. Oral habits, prevalence and effects on occlusion of 4-15 year old school children in Lagos, Nigeria. Niger Postgrad Med J. 2010; 17 (2): 113-7.

30. Ize-Iyamu IN, Isiekwe MC. Prevalence and factors associated with anterior open bite in 2 to 5 year old children in Benin city, Nigeria. Afr Health Sci. 2012; 12 (4): 446-451 